\title{
Technosciences et innovations citoyennes
}

\section{Technosciences and citizen innovations}

\author{
Jean-Claude Ruano-Borbalan ${ }^{1}$, Bertrand Bocquet ${ }^{2}$
}

${ }^{1}$ Laboratoire Histoire des Technosciences en Société, CNAM, Paris, France, jean-claude.ruanoborbalan@lecnam.net
${ }^{2}$ Laboratoire Histoire des Technosciences en Société, CNAM, Université de Lille, France, Bertrand.Bocquet@univ-lille.fr

RÉSUMÉ. Ce dernier demi-siècle a vu un développement considérable d'interfaces institutionnelles participant à la « grande standardisation » des systèmes scientifiques et d'innovation. Les limites de ce modèle sont apparues pour de multiples raisons, aussi bien économique que politique ou culturel. De fortes évolutions s'inscrivent dans le contexte d'une démocratie plus délibérative qui impacte nécessairement la production et les institutions scientifiques ou techniques et bien entendu la nature et les politiques de l'innovation. L'interrogation est donc de savoir si ce mouvement relevant d'une «démocratie technique» va s'installer durablement. Nous consacrons ce numéro aux innovations citoyennes, participatives, liées peu ou prou aux questions techniques et scientifiques. II met en avant des échelles et focales diverses dans ces domaines de « l'innovation sociale et citoyenne » en s'appuyant sur des exemples de transformations en cours.

ABSTRACT. The last half century has seen considerable development of institutional interfaces participating in the "great standardization" of science and innovation systems. The limitations of this model appeared for many economic, political or cultural reasons. Strong developments appear within the context of a deliberative democracy that impacts scientific and technical institutions and production, and therefore the nature and the policies of innovation. The question about this part of a "technical democracy" is whether there will be a long-term movement. We dedicate this issue to citizen participatory innovations, more or less related to technical and scientific questions. It highlights various scales and focal points of "social and citizen innovation", domains based on examples of ongoing transformations.

MOTS-CLÉS. Recherche Action Participative, Innovation sociale, Controverse, Expertise collective, Capacitation, Société de la connaissance, Co-construction, Co-production des savoirs, Sciences en société, Démocratie technique.

KEYWORDS. Participatory Action Research, Social innovation, Controversy, Collective expertise, Empowerment, Knowledge society, Co-construction, Co-production of knowledge, Sciences in society, Technical democracy.

Ce dernier demi-siècle a vu un développement considérable d'interfaces institutionnelles, publiques ou privées, avec la sphère technoscientifique et industrielle, notamment au travers de la mise en place dans les principaux pays de l'OCDE de puissantes politiques publiques de recherche et d'innovation. Ce mouvement s'est accompagné d'une optimisation grandissante des procédures de production de la connaissance scientifique, des définitions disciplinaires (nomenclature Frascati de l'OCDE par exemple), ainsi que des « produits » issus des activités scientifiques ou techno-scientifiques : articles, colloques ou brevets. Cette «grande standardisation» de la science et des techno-sciences est passée par une internationalisation et une comparabilité (évaluation de plus en plus prégnante). Elle a pour but explicite de garantir une meilleure efficacité des processus de transferts de connaissances vers des activités économiquement solvables et cherchant une « utilité industrielle » forte. Cette optimisation et rationalisation des systèmes scientifiques et d'innovation fait l'objet d'un nombre croissant de recherches tant pour en comprendre l'ampleur et en améliorer l'analyse que pour proposer des modélisations susceptibles d'être transposées.

Aujourd'hui donc, l'OCDE comme l'Union Européenne ou les divers ministères de la recherche et de l'industrie et enfin les villes et gouvernements territoriaux, promeuvent des « système nationaux de recherche et d'innovation» extrêmement sophistiqués, destinés dans la rhétorique européenne par exemple, à répondre aux «défis» contemporains et selon toutes les institutions à développer la croissance économique. Ces institutions et les politiques publiques instaurées ont accompagné la mise en ouvre d'une diversité d'outils et de dispositifs allant du transfert de technologie à la création de pépinières d'entreprises, de la gestion de plus en plus complexe de portefeuilles de propriétés intellectuelles jusqu'au soutien direct et organisé à la créativité entrepreneuriale. Ces politiques et 
institutions ont eu des effets massifs et généralisés, en termes de développement technologique ou industriel mais restant essentiellement sous la forme de processus d'innovations linéaires, aussi bien industrielles que de gestion.

Durant les années 1980 à 2000, les limites de ce modèle de promotion de l'innovation industrielle et sociale reposant sur le développement des technosciences et leurs applications directes dans l'industrie ou les services sont apparues et se sont renforcées pour de multiples raisons, qui peuvent être analysées du point de vue culturel ou politique autant que du point de vue économique. On constate en effet depuis un demi-siècle environ, des mutations idéologiques et politiques liées à l'approfondissement de l'individualisation dans la «post-modernité», renforçant les sensibilités aux risques de toutes natures (santé, environnement, etc.). On assiste également et conjointement à des transformations dans les formes de la mobilisation politique et sociale (conception du bien commun et de la démocratie par exemple), etc. L'ensemble des mutations politico-idéologiques a rencontré et renforcé la prise de conscience de la faible prise en compte des «externalités négatives» des techno-sciences et des politiques industrielles reliées, par le modèle d'innovation et de développement techno-scientifique ou économique dominant. Ces externalités prennent une dimension inconnue jusqu'ici et qui, pour les opposants au modèle de l'innovation techno-scientifique et industriel linéaire peuvent concerner jusqu'aux conditions même de l'existence de l'humanité sur terre : dégradation de l'environnement, changement climatique, problèmes sanitaires, mais aussi sociaux ou politiques si l'on songe simplement à l'hypothèse désormais commune du risque de migrations de réfugiés climatiques par exemple.

La crise démocratique des sociétés contemporaines n'est pas uniquement liée aux questions soulevées par l'innovation et les techno-sciences, mais elle trouve là une expression et des formes de réflexions importantes et reconnues. On constate des évolutions fortes, dont les plus marquantes et novatrices s'inscrivent dans le contexte d'une démocratie plus délibérative et «participative». En retour, et par un phénomène de boucle de rétro-action, ce mouvement de réappropriation impacte nécessairement la production et les institutions scientifiques ou techniques et bien entendu la nature et les politiques de l'innovation.

L'interrogation est donc de savoir, entre autres, si ce mouvement de développement de l'implication des acteurs sociaux et de la société civile (associations, ONG) dans des formes de mobilisations et d'expertises caractérisé depuis la fin des années 2000 comme relevant d'une «démocratie technique» va s'installer durablement. Est-il capable de produire des connaissances nouvelles, issues de l'interaction entre des acteurs scientifiques, des pouvoirs publics et des expertises et participation de la société civile ? Ces expériences et dispositifs de participation et d'interfaces entre sciences, pouvoirs publics et citoyens sont-ils efficaces pour la résolution des grands défis sociétaux, notamment liés aux «transitions» énergétiques, à l'urbanisation, etc. C'est l'objet de numéro que de contribuer à éclairer ce type d'interrogation à partir de cas concrets d'expérimentation. Le propos est de regarder de plus près les lieux d'interfaçage entre les techno-sciences et les acteurs de la société civile ou groupements d'acteurs-citoyens, naissant pour l'essentiel de la volonté d'ouverture des politiques publiques à la participation et l'innovation sociale, qui caractérise ce que la philosophie politique occidentale caractérise comme le «deliberative turn», depuis presque un demi siècle.

Une emphase particulière est portée dans cette livraison sur la notion de «sciences et recherches participatives» qui constituent actuellement un domaine d'interrogations en croissance, pris en compte dans une certaine mesure par les États, autour d'un foisonnement d'expériences. Ce mouvement, initié plutôt en Amérique du Nord ou Europe est «métabolisé» pour de multiples raisons par de grands organismes de recherche comme l'INRA en France. Le président de cet organisme (François Houllier) a été chargé (en 2016) par le gouvernement français de rédiger un rapport sur ce sujet, mettant en avant une vision faible et une vision forte des sciences participatives : soit comme auxiliaire passives des organismes et acteurs scientifiques, soit comme acteur plein contribuant réellement à la production de connaissance et de modèles d'action partagés. Les «sciences participatives» ont bénéficié de dispositifs 
variés pour susciter le dialogue, la délibération ou même parfois, selon un vocabulaire plus récent issu des travaux du sociologue Michel Callon : la «co-construction des savoirs». Des questionnements liés à des problématiques posées par la société civile peuvent ouvrir le monde de la recherche à des sujets davantage ancrés dans la complexité des interactions politiques et sociales.

C'est ainsi que le ministère de l'Écologie, du Développement Durable et de l'Énergie français a créée le programme REPERE (Réseau d'échange et de projets sur le pilotage de la recherche et de l'expertise) entre 2009 et 2013. Ce programme reposait sur la participation des associations à la discussion pour réformer le pilotage de la recherche et de l'expertise sur les thématiques du développement durable, avec les quatre dimensions affichées suivantes: (i) impliquer les parties prenantes dans l'orientation de la recherche; (ii) faciliter les démarches d'innovation avec tous les acteurs ; (iii) associer les parties prenantes à une production efficace de données et de connaissances ; (iv) faciliter et favoriser l'accès aux données et aux résultats scientifiques. En 2012, la mission du CNRS « Sciences et citoyens » contribue à formaliser la notion de recherche coopérative tandis que la mission actuelle «Sciences en société » élargit le domaine d'intervention du CNRS à l'InSHS. En janvier 2014, c'est au sein de l'Institut Écologie et Environnement (INEE) que se crée le Groupement de Recherche du CNRS intitulé « Participatory Action Research and Citizen Science » (GDR PARCS) dont la vocation est de faire émerger une communauté de recherche centré sur le concept de Recherche Action Participative (RAP). Le rapport du président de l'INRA sus-nommé met bien en évidence une diversité d'expériences et d'acteurs en sciences et recherches participatives. Du côté de la société civile, cette mise en évidence avait commencé par une étude de l'association Sciences Citoyennes, financée en 2013 par la Fondation de France, qui révélait un «continent invisible» de deux cents expériences de recherches participatives et le colloque-forum organisé par l'ALLISS (Alliance Sciences-Société) organisé en janvier 2015 mit à jour plus de quatre cents projets de recherche acteurs-chercheurs en France. Notons que dans Horizon 2020, ces rapprochements bénéficient du programme transversal « Sciences avec et pour la société ». Ce courant participatif avait touché depuis longtemps l'Amérique du Nord avec les «Community-Based Research» aux USA ou le programme ARUC (Alliance de Recherche Université - Communauté) au Canada. Ces derniers ont inspiré les programmes régionaux français : «Programme Institution Citoyen de Recherche et d'Innovation » (PICRI) en Île-de-France créé en 2006, «Appropriation Sociale des Sciences » (ASoSc) en Bretagne en 2009, « Chercheurs Citoyens » en Nord-Pas de Calais en 2011.

Dans ce contexte d'évolution des rapports sciences-société où, dans les principaux pays industrialisés, la question de «l'expertise» des acteurs de la société civile ou des acteurs-citoyens, est pointée. Plus généralement, et en lien - mais pas uniquement, si l'on note le cas de la Chine- avec une mutation des cadres et fondements de la légitimité démocratique ou de l'action publique, la «participation» citoyenne est devenu une préoccupation importante, et des traductions institutionnelles fortes se formalisent, tant au sein de dispositifs de concertation, visant souvent plus à l'acceptabilité des politiques publiques qu'au développement d'une «démocratie technique». Ces processus de légitimation et de définition des politiques publiques s'observent particulièrement dans le cadre des rapports sciences-société et les politiques d'aménagement urbains ou de développement des écosystèmes d'innovation. C'est le cas par exemple en France de la loi sur l'Enseignement Supérieur et la Recherche de juillet 2013, qui institutionnalise une troisième mission des universités orientée vers ces questions de rapports sciences-société. Celle-ci traduit la volonté d'une meilleure prise en compte des questions concernant la société et s'inscrit aussi dans la Stratégie Nationale de Recherche et ses 10 défis sociétaux ${ }^{1}$. D'autres déclinaisons se font jour dans d'autres secteurs où la recherche scientifique n'est pas la fonction principale : par exemple la loi sur la transition énergétique d'août 2015 qui préconise d'associer les différents acteurs (institutions et acteurs scientifiques, associations,individus) en y adjoignant les représentants des politiques publiques territoriales.

\footnotetext{
${ }^{1} \mathrm{http} / /$ www.enseignementsup-recherche.gouv.fr/cid78733/strategie-nationale-de-recherche-dix-grands-defis-societaux.html 
En conséquence, si nous avons assisté à un élargissement sans précédent des discours et pratiques prônant l'innovation techno-industrielle ou managériale dans le demi-siècle écoulé, nous voyons également apparaître la convergence de préoccupations politiques à toutes les échelles de la gouvernance, mais aussi associatives, prônant l'avènement de nouvelles formes de développement économique et d'innovation, passant pour l'essentiel par l'accroissement de la participation et de «l'encapacitation» (empowerment) des acteurs sociaux et politiques. L'affirmation de ces nouvelles postures et formes plus diffuses de l'innovation, qualifiée de «sociale» ou de «citoyenne» est présente au travers des tissus des organisations de la société civile. Elle peut se manifester au travers de mobilisations citoyennes sur des controverses techno-scientifiques par exemple mais pas seulement. En effet, un mouvement général plus volontariste de réappropriation de la question de l'innovation se fait jour dans la volonté des sciences et recherches participatives, dans les perspectives de pédagogies universitaires ou autres renouvelées, etc. Ces formes sont vécues par les acteurs comme plutôt « ascendantes ». Elles rencontrent et irriguent depuis deux ou trois décennies la volonté d'amélioration des fonctionnements administratifs ou politiques de la puissance publique. Des formes plus élaborées sont même envisagées, basées sur la coopération, la production collaborative (co-production) de connaissance et de travail, des dispositifs visant à la pluridisciplinarité, etc. Il est à noter que ces discours et dispositifs prônant la capacité des individus et collectifs, s'incarnent également au sein de « tiers lieux » comme les fab-labs, les living labs, les boutiques des sciences, dans le mouvement des « makers », dans la prise en compte des usagers et de leurs capacités cognitives, dans le «co-design» ou la «maîtrise d'usage», etc. Dans l'ensemble de ces lieux nouveaux, formes de mobilisation sociale ou politique, la participation et la coopération, mais aussi des méthodes nouvelles de délibération ou construction des argumentations, issues du design et de l'innovation sociale ou pédagogique, sont mises en avant, au sein de multiples formes organisationnelles où les technologies de la communication jouent un rôle de catalyseur. On constate qu'en ces multiples lieux, les frontières entre les traditions associatives multiples et les nouvelles formes d'engagement, y compris entrepreneuriaux, sont ténues, voire inexistantes.

Le présent numéro de la revue Technologie et innovation est consacré aux innovations citoyennes, participatives, liées peu ou prou aux questions techniques et scientifiques. Il met en avant des échelles et focales diverses et fournit des exemples de transformations en cours, dans ces domaines de «l'innovation sociale et citoyenne». Cette livraison interroge ainsi particulièrement la question du «sens» de l'innovation et du développement techno-scientifique : l'une des interrogations aujourd'hui en pointe dans plusieurs disciplines, de la sociologie politique aux Sciences and technologies studies, en passant par l'analyse organisationnelle ou les sciences de gestion.

Jean Claude Ruano-Borbalan s'interroge sur la Démocratie technique et ses transformations. Il pointe l'écart insurmontable existant entre un idéal démocratique d'implication et de participation citoyenne, exprimé dans des formes contemporaines de développement de formes collaboratives et participatives de la délibération, et la réalité contradictoire de biais et contraintes fortes pesant sur les processus et dispositifs de participation et délibération, notamment et principalement dans le cas des rapports sciences, technosciences et société, communément désignés sous le vocable de « démocratie technique $\gg$.

Saliha Hadna, dans son article, invite à penser la co-production des connaissances en dehors des «forums hybrides», au travers de la controverse menée autour du recensement des stériles de mines d'uranium. Elle s'intéresse à la problématique du suivi environnemental, grâce à l'étude de l'ancienne mine de Pen Ar Ran (Loire-Atlantique) et montre que si aucune instance de concertation avec les populations n'a été mise en place, un groupe de citoyens s'est organisé pour contribuer à la décontamination de certaines zones impactées par la radioactivité des stériles miniers. L'intérêt est là d'analyser la trajectoire empruntée par cette cause collective ainsi que les moyens mobilisés par les acteurs sociaux/citoyens dans la production des connaissances liées à la radioactivité. En particulier, elle montre que l'acquisition de moyens de mesure et la formation à leur utilisation permet à ce collectif de développer un argumentaire fondé sur des bases scientifiques. 
Antonella Tufano dans un article sur l'ampleur et les limites du discours participatif pour la gouvernance et la planification territoriale, montre, elle, que l'innovation est aujourd'hui la modalité de développement des projets à l'échelle de la ville et des territoires. Depuis plus de vingt ans, souligne l'auteure, sous l'influence d'intellectuels, la planification urbaine et territoriale a dû se questionner au regard des questions sociales et intégrer une forme d'ouverture grâce à la participation. Ce questionnement a généré des synthèses innovation sociale/innovation territoriale qui n'ont pas été questionnées, et qui montre-t-elle prennent peu en compte les possibles solutions émergentes des actions participatives. L'analyse proposée par Antonella Tufano invite à regarder si (et comment) l'introduction de visées participatives dans la démarche de planification permet ou non, d'implémenter l'innovation sociale et celle d'ordre technoscientifique liées aux infrastructures dans les espaces territoriaux.

En contrepoint de ces approches mettant en lumière plutôt les biais et contraintes du «tournant participatif», Sylvie Blangy, Bertrand Bocquet et leurs co-auteurs proposent une méthodologie et une approche transversale dite de Recherche Action Participative. Au-delà de la prise en compte de savoirs issus des sciences expérimentales, sociales et humaines, la méthodologie cherche à embrasser les problématiques citoyennes directement en prise avec les défis environnementaux, technoscientifiques, et autres. La Recherche Action Participative permet selon les auteurs de développer des méthodologies de recherche spécifiques liant chercheurs, représentants associatifs, praticiens, etc. Les auteurs s'appuient notamment sur l'expérience menée par le GDR CNRS PARCS dont le fonctionnement peut s'apparenter à un laboratoire de recherche «hors-mur». Ils examinent l'impact qu'il a eu sur ses membres notamment au travers de cycles de formation et les conclusions positives qui en sont issues.

Les sciences participatives, traduction française de «citizen science», constituent l'un des lieux prometteurs, mais aussi fort contrasté, de la rencontre entre sciences, acteurs/citoyens et politiques publiques ou industrielles de recherche. Elles s'appuient sur une large mobilisation citoyenne, le développement du numérique et de nouvelles formes d'interaction science-société. Sylvie Blangy et d'autres co-auteurs, examinent cette question, dont plusieurs grands organismes de recherche comme le MNHN ou l'INRA depuis une dizaine d'années proposent le développement. L'interrogation porte sur sur la synergie entre les équipes de recherche et les citoyens «collecteurs» de données notamment sur les phases d'analyse des données. En effet, les citoyens sont cantonnés dans cette phase de production de données au sein de protocoles définis par les chercheurs. Les auteurs plaident pour que les méthodologies déployées au sein des sciences citoyennes se fassent au-delà de la collecte des données pour qu'elles permettent d'ouvrir le champ du questionnement de recherche en amont et celui de l'analyse et de l'interprétation des données en aval aux citoyens.

En complément, Orelie Desfriches Doria et ses co-auteurs, présentent une réflexion sur le rôle de la maîtrise de l'information dans l'analyse et la médiation de controverse socio-scientifique ou sociotechnique, considérée comme un dispositif social innovant. L'article s'appuie sur une méthodologie d'analyse des controverses liée au contexte informationnel complexe actuel. Il développe la méthodologie originale produite par les auteurs et synthétise les principaux résultats préliminaires de sa mise à l'épreuve auprès d'un public étudiant depuis 2014.

Marie Goyon montre, grâce à une étude sur les formes d'innovation pédagogique, le rôle potentiel de nouvelles formes de production coopératives issues du mouvement des «Fab-Lab» dans la transformation du métier d'ingénieur. L'article propose de réfléchir en particulier à l'impact sur le métier d'ingénieur généraliste. Ouvrant à des dynamiques de co-construction et de communication entre acteurs hétérogènes (scientifiques, ingénieurs, citoyens, travailleurs). En associant «bricolage» et « rétro-ingénierie », ces environnements Fab-Labs semblent pouvoir renouveler les démarches de conception, voire même la culture et le métier d'ingénieur. Le cadre du paradigme actuel de «l'innovation collaborative» est entendue comme un appel à "décloisonner", à sortir des « frontières » pédagogiques, disciplinaires, territoriales et managériales entre les espaces des écoles, de la recherche, de l'industrie, de la société civile. 
Pour finir, Davia Dosias-Perla et ses co-auteurs présentent les premiers résultats d'une rechercheaction menée à partir d'un dispositif original «Artivistes-atelier » en recherche action participative portant sur la qualité de l'air extérieur sur le territoire de Montpellier. En détaillant le design élaboré, l'article décrit la construction d'une cartographie dynamique des acteurs impliqués/impactés et leur capacité d'action. Pour cela l'article décrit cette expérience de terrain à la fois militante, réflexive et critique en tant que dispositif relevant de l'innovation sociale issu du milieu associatif (Artivistesatelier) dialoguant avec des chercheurs, issus du Groupe de Recherche PARCS (Participatory Action Research and Citizen Sciences) portant plus spécifiquement sur les problématiques socioenvironnementales. Le dispositif «Artivistes-atelier» disent les auteurs, vise d'une part à décloisonner les enjeux socio-environnementaux et d'autre part à accompagner l'application des politiques publiques sur les territoires. Le dispositif agit comme un marqueur des potentialités de mobilisation citoyenne, de la démocratie participative mais aussi du monde de la recherche et de différentes structures institutionnelles ou non au carrefour sciences-sociétés.

Le numéro « technosciences et innovation citoyenne» invite donc à s'interroger sur l'ampleur et les limites nettes des dispositifs de participation et d'interfaces entre acteurs sociaux/citoyens ; discours scientifique et engagement de chercheurs; réalité et objectifs des politiques publiques. Il rappelle que la question des dispositif est centrale, quoique sa pensée soit bien plus faible que la réflexion générale sur les gains et nécessités supposées d'une approche et de discours prônant l'implication et la participation citoyenne. Il invite à un scepticisme mesuré, à se souvenir peut-être, pour ceux qui militent et se sentent engagés dans le développement de formes de démocratie technique ou de participation citoyenne, fusse au nom de l'analyse «scientifique» de la force de la maxime attribuée à Gramsci : « Il faut allier le pessimisme de l'intelligence à l'optimisme de la volonté ». 